PROCEEDINGS OF THE

AMERICAN MATHEMATICAL SOCIETY

Volume 127, Number 10, Pages 2899-2904

S 0002-9939(99)05266-1

Article electronically published on April 23, 1999

\title{
FURTHER EXTENSION OF THE HEINZ-KATO-FURUTA INEQUALITY
}

\author{
MITSURU UCHIYAMA
}

(Communicated by David R. Larson)

\begin{abstract}
Let $T$ be a bounded operator on a Hilbert space $\mathfrak{H}$, and $A, B$ positive definite operators. Kato has shown that if $\|T x\| \leq\|A x\|$ and $\left\|T^{*} y\right\| \leq$ $\|B y\|$ for all $x, y \in \mathfrak{H}$, then $|(T x, y)| \leq\|f(A) x\|\|g(B) y\|$, where $f(t), g(t)$ are operator monotone functions defined on $[0, \infty)$ such that $f(t) g(t)=t$. Furuta has shown that $\left|\left(T|T|^{\alpha+\beta-1} x, y\right)\right| \leq\left\|A^{\alpha} x\right\|\left\|B^{\beta} y\right\|$, where $0 \leq \alpha, \beta \leq 1,1 \leq$ $\alpha+\beta$. Let $f(t), g(t)$ be any continuous operator monotone functions, and set $h(t)=f(t) g(t) / t$ for $t>0$. We will show that $T h(|T|)$ is well defined and $|(T h(|T|) x, y)| \leq\|f(A) x\|\|g(B) y\|$. Moreover, we will extend this result for unbounded closed operators densely defined on $\mathfrak{H}$.
\end{abstract}

\section{INTRODUCTION}

Let $B(\mathfrak{H})$ be the $C^{*}$-algebra of all bounded operators on a Hilbert space $\mathfrak{H}$. Let $f$ be a real valued continuous function defined on $[0, \infty)$. If $f$ satisfies $f(A) \leq f(B)$ whenever $0 \leq A \leq B, A, B \in B(\mathfrak{H})$, then $f$ is called an operator monotone function. $f$ is an operator monotone function if and only if $f$ has an analytic extension that is holomorphic on the upper half plane and maps the upper half plane into it [4]. Therefore, if $0 \leq f(t)$ is an operator monotone function on $[0, \infty)$, then so is $f(\sqrt{t})^{2}$. This implies that for $A, B \geq 0$,

$$
\|A x\| \leq\|B x\| \text { for every } x \in \mathfrak{H} \rightarrow\|f(A) x\| \leq\|f(B) x\| \text { for every } x \in \mathfrak{H} .
$$

$t^{\alpha}(0<\alpha \leq 1)$ and $\log (1+t)$ are operator monotone functions [2], [4]. Kato has shown the following.

Theorem A ([3]). Let $T$ be a bounded operator on a Hilbert space $\mathfrak{H}$, and $A, B$ positive definite operators. If $\|T x\| \leq\|A x\|$ and $\left\|T^{*} y\right\| \leq\|B y\|$ for all $x, y \in \mathfrak{H}$, then

$$
|(T x, y)| \leq\left\|A^{\alpha} x\right\|\left\|B^{1-\alpha} y\right\| \quad \text { for any } \quad \alpha \in[0,1] .
$$

Actually, he has shown that the above inequality holds for (unbounded) closed operators $T, A, B$, and furthermore that $A^{\alpha}$ and $B^{1-\alpha}$ in the above inequality can

Received by the editors June 6, 1997 and, in revised form, December 9, 1997.

1991 Mathematics Subject Classification. Primary 47A63, 47A30, 47B25.

Key words and phrases. Löwner theory, Heinz inequality, operator monotone function, closed operator, non-negative operator.

This research was partially supported by Grant-in-Aid for Scientific Research.

(C)1999 American Mathematical Society 
be replaced by $f(A)$ and $g(B)$, where $f(t), g(t)$ are operator monotone functions defined on $[0, \infty)$ such that $f(t) g(t)=t$, that is,

$$
|(T x, y)| \leq\|f(A) x\|\|g(B) y\| .
$$

Furuta has extended (2) as follows.

Theorem B ([1]). Let $T$ be a bounded operator on a Hilbert space $\mathfrak{H}$, and $A, B$ positive definite operators. If $\|T x\| \leq\|A x\|,\left\|T^{*} y\right\| \leq\|B y\|$ for all $x, y \in \mathfrak{H}$, then

$$
\left|\left(T|T|^{\alpha+\beta-1} x, y\right)\right| \leq\left\|A^{\alpha} x\right\|\left\|B^{\beta} y\right\|,
$$

where $0 \leq \alpha, \beta \leq 1,1 \leq \alpha+\beta$.

The aim of this paper is to extend (3) and (4). Actually, we will show that $T((f g) / t)(|T|)$ is well defined for every operator monotone function $f(t), g(t)$ and

$$
|(T((f g) / t)(|T|) x, y)| \leq\|f(A) x\|\|g(B) y\| .
$$

We will treat bounded operators in the next section and unbounded closed operators in the last section.

\section{BOUNDED OPERATORS}

Lemma 1. Let $A=\int_{-0}^{\infty} \lambda d E_{\lambda}$ be the spectral decomposition of $A \geq 0$. Then $A(A+\delta)^{-1}$ strongly converges to $I-E_{0}$ as $\delta \rightarrow+0$.

Proof. $A(A+\delta)^{-1} E_{0} x=(A+\delta)^{-1} A E_{0} x=0$ for $x \in \mathfrak{H}$. Thus we have

$$
\begin{aligned}
& \left\|A(A+\delta)^{-1} x-\left(I-E_{0}\right) x\right\|^{2}=\left\|\left\{A(A+\delta)^{-1}-I\right\}\left(I-E_{0}\right) x\right\|^{2} \\
& =\int_{-0}^{\infty}\left|\frac{\lambda}{\lambda+\delta}-1\right|^{2} d\left\|\left.E_{\lambda}\left(I-E_{0}\right) x\right|^{2}=\int_{+0}^{\infty}\left|\frac{\delta}{\lambda+\delta}\right|^{2} d\right\| E_{\lambda}\left(I-E_{0}\right) x \|^{2} .
\end{aligned}
$$

From Lebesgue's dominated convergence theorem, this converges to 0 as $\delta \rightarrow+0$.

Lemma 2. Let $T=V|T|$ be the polar decomposition of $T$. Then

$$
T(|T|+\delta)^{-1} \rightarrow V \text { (strongly) as } \delta \rightarrow+0 .
$$

Proof. Let $\left\{E_{\lambda}\right\}$ be the spectral family for $|T|$. By Lemma $1,|T|(|T|+\delta)^{-1} \rightarrow I-E_{0}$ as $\delta \rightarrow+0$. Thus we have

$$
T(|T|+\delta)^{-1} \rightarrow V\left(I-E_{0}\right) \text { (strongly) as } \delta \rightarrow+0 .
$$

Since $E_{0}$ is the projection onto the eigen-space $\{x: T x=0\}$, we get $V E_{0}=0$. Consequently,

$$
T(|T|+\delta)^{-1} \rightarrow V \text { (strongly) as } \delta \rightarrow+0 .
$$

Definition 1. For a function $h(t)$ we set $h_{\delta}(t)=h(t+\delta)$.

Lemma 3. Let $h(t)$ be a continuous function defined on $(0, \infty)$. If th(t) has the continuous extension $f(t)$ on $[0, \infty)$, then

$$
T h_{\delta}(|T|) \rightarrow V f(|T|) \text { (strongly) as } \delta \rightarrow+0 .
$$

Proof. We note that $T h_{\delta}(|T|)=T(|T|+\delta)^{-1} f(|T|+\delta)$. Since $f(|T|+\delta)$ converges to $f(|T|)$ in the norm toplogy, from Lemma 2 it follows that $T h_{\delta}(|T|) \rightarrow V f(|T|)$ (strongly) as $\delta \rightarrow+0$. 
Definition 2. Under the same assumption as in Lemma 3, we denote the limit of $T h_{\delta}(|T|)$ as $\delta \rightarrow+0$ by $T h(|T|)$, which is equal to $V f(|T|)$.

Let us remark that we defined not $h(|T|)$, but $T h(|T|)$. If $h(t)$ is continuous on $[0, \infty)$, then $h(|T|)$ is well defined, and the product of $T$ and $h(|T|)$ is coincident with $T h(|T|)$ defined above. It is clear that if $h(t)=t^{-\alpha}(0<\alpha \leq 1)$, then $T h(|T|)$ is well defined and $T h(|T|)=V|T|^{1-\alpha}$.

Theorem 4. Let $f$ and $g$ be any continuous operator monotone functions defined on $[0, \infty)$. Then $T((f g) / t)(|T|)$ is well defined for every $T$ in $B(\mathfrak{H})$ in the sense of Definition 2. Moreover, if

$$
\|T x\| \leq\|A x\|,\left\|T^{*} y\right\| \leq\|B y\| \quad \text { for all } \quad x, y \in \mathfrak{H},
$$

where $A, B \geq 0$, then

$$
|(T((f g) / t)(|T|) x, y)| \leq\|f(A) x\|\|g(B) y\| \quad \text { for all } \quad x, y \in \mathfrak{H} .
$$

Proof. Set $h(t)=f(t) g(t) / t$ for $t>0$. It is clear that $t h(t)$ is continuous on $[0, \infty)$ and hence $T h(|T|)=T((f g) / t)(|T|)$ is well defined. Let $T=V|T|$ be the polar decomposition of $T$. Then by Lemma 3 we obtain

$$
\begin{aligned}
T h(|T|) & =V(f g)(|T|)=V f(|T|) g(|T|), \\
|(T h(|T|) x, y)| & =|(V f(|T|) g(|T|) x, y)|=\left|\left(g\left(\left|T^{*}\right|\right) V f(|T|) x, y\right)\right| \\
& \leq\|f(|T|) x\|\left\|g\left(\left|T^{*}\right|\right) y\right\|,
\end{aligned}
$$

and by (1)

$$
\leq\|f(A) x\|\|g(B) y\|
$$

We remark that (5) is an extension of both (3) and (4): in fact, we removed the conditions $f(t) g(t)=t$ and $1 \leq \alpha+\beta$ from (3) and (4), respectively.

\section{UNBOUNDED CLOSED OPERATORS}

From now on, we consider the case where $T, A, B$ are unbounded closed operators densely defined on $\mathfrak{H}$. Let us remember that for a self-adjoint operator $A$ and for continuous functions $f, g, f(A) g(A) \subseteq(f g)(A)$ and that if $x \in \mathfrak{D}(g(A)) \cap$ $\mathfrak{D}((f g)(A))$, then $x \in \mathfrak{D}(f(A) g(A))$ and $f(A) g(A) x=(f g)(A) x$.

It is known [4], [5] that an operator monotone function $f$ on $[0, \infty)$ has an integral representation

$$
f(t)=\alpha+\beta t-\int_{0}^{\infty}\left(\frac{1}{t+s}-\frac{s}{s^{2}+1}\right) d v(s) \text { for } t>0
$$

where $\alpha$ and $\beta$ are real, $\beta \geq 0$, and $v$ is a non-negative Borel measure such that

$$
\int_{0}^{\infty} \frac{1}{1+s^{2}} d v(s)<\infty
$$

Since

$$
\lim _{t \rightarrow+0} f(t)=f(0) \text { and } \lim _{t \rightarrow+0} \int_{\frac{1}{t}}^{\infty}\left(\frac{s}{s^{2}+1}-\frac{1}{t+s}\right) d v(s)=0
$$


it follows that

$$
\begin{aligned}
f(0) & =\alpha-\lim _{t \rightarrow+0} \int_{0}^{\frac{1}{t}}\left(\frac{1}{t+s}-\frac{s}{s^{2}+1}\right) d v(s) \\
& =\alpha-\lim _{t \rightarrow+0} \int_{0}^{\infty}\left(\frac{1}{t+s}-\frac{s}{s^{2}+1}\right) \chi_{\left[0, \frac{1}{t}\right]} d v(s) .
\end{aligned}
$$

From Fatou's theorem it follows that

$$
\int_{0}^{\infty}\left(\frac{1}{s}-\frac{s}{s^{2}+1}\right) d v(s)<\infty, \text { and hence } f(0)=\alpha-\int_{0}^{\infty}\left(\frac{1}{s}-\frac{s}{s^{2}+1}\right) d v(s) .
$$

Thus we have

$$
f(t)=f(0)+\beta t+\int_{0}^{\infty}\left(\frac{1}{s}-\frac{1}{t+s}\right) d v(s) .
$$

Since $f(1)<\infty$, we get

$$
\int_{0}^{\infty} \frac{1}{s(1+s)} d v(s)<\infty
$$

Lemma 5. Let $0 \leq f(t), g(t)$ be operator monotone functions, and let $A \geq 0$ be an unbounded self-adjoint operator. Then $\mathfrak{D}(A) \subseteq \mathfrak{D}(f(A))$, where $\mathfrak{D}(A)$ stands for the domain of $A$. Moreover, $\mathfrak{D}\left(A^{2}\right) \subseteq \mathfrak{D}(f(A) g(A)) \subseteq \mathfrak{D}((f g)(A))$.

Proof. By (7) and (8), it is clear that $f(t) / t$ and $g(t) / t$ are bounded on $[1, \infty)$. Hence we can see

$$
\mathfrak{D}(A) \subseteq \mathfrak{D}(f(A)) \quad \text { and } \quad \mathfrak{D}(A) \subseteq \mathfrak{D}(g(A)) .
$$

Similarly, from the boundedness of $f(t) g(t) / t^{2}$ on $[1, \infty)$ it follows that $\mathfrak{D}\left(A^{2}\right) \subseteq$ $\mathfrak{D}((f g)(A))$. Thus for an arbitrary $x \in \mathfrak{D}\left(A^{2}\right)$, we have

$$
x \in \mathfrak{D}(g(A)) \quad \text { and } \quad x \in \mathfrak{D}((f g)(A)),
$$

and hence

$$
x \in \mathfrak{D}(f(A) g(A)) \quad \text { and } \quad f(A) g(A) x=(f g)(A) x .
$$

Proposition 6. Let $T$ be a densely defined closed operator, and let $f$ and $g$ be arbitrary operator monotone functions defined on $[0, \infty)$, such that $f \geq 0, g \geq 0$. Set $h(t)=((f g) / t)$ for $t>0$. Then

$$
\begin{gathered}
\mathfrak{D}\left(T h_{\delta}(|T|)\right)=\mathfrak{D}((f g)(|T|)) \text { for every } \delta>0 \\
T h_{\delta}(|T|) x \rightarrow V(f g)(|T|) x \quad(s) \quad \text { as } \quad \delta \rightarrow+0 \text { for } x \in \mathfrak{D}((f g)(|T|)) .
\end{gathered}
$$

Proof. By (7),

$$
0 \leq f(t+\delta)-f(t) \leq f(\delta)-f(0), \quad 0 \leq g(t+\delta)-g(t) \leq g(\delta)-g(0) .
$$

This implies

$$
\begin{aligned}
1 \leq \frac{f(t+\delta)}{f(t)} \frac{g(t+\delta)}{g(t)} & \leq\left(1+\frac{f(\delta)-f(0)}{f(t)}\right)\left(1+\frac{g(\delta)-g(0)}{g(t)}\right) \\
& \leq\left(1+\frac{f(\delta)-f(0)}{f(1)}\right)\left(1+\frac{g(\delta)-g(0)}{g(1)}\right) \quad(t>1) .
\end{aligned}
$$


Thus we can see that

$$
\mathfrak{D}((f g)(|T|+\delta))=\mathfrak{D}((f g)(|T|)),
$$

$$
(f g)(|T|+\delta) x \rightarrow(f g)(|T|) x \quad(s) \quad(\delta \rightarrow+0) \text { for } x \in \mathfrak{D}((f g)(|T|)) .
$$

By $(f g)(t+\delta) \geq \delta h(t+\delta)$, we obtain

$$
\mathfrak{D}((f g)(|T|+\delta)) \subseteq \mathfrak{D}(h(|T|+\delta)) .
$$

Therefore, using $(|T|+\delta)(h(|T|+\delta)) \subseteq(f g)(|T|+\delta)$ we get

$$
(|T|+\delta)(h(|T|+\delta))=(f g)(|T|+\delta) .
$$

Thus we obtain

$$
\left.T h_{\delta}(|T|)=\left\{V|T|(|T|+\delta)^{-1}(|T|+\delta)\right\} h_{\delta}(|T|)=\left\{V|T|(|T|+\delta)^{-1}\right\}(f g)(|T|+\delta)\right) ;
$$

here we used $\mathfrak{D}(|T| h(|T|+\delta))=\mathfrak{D}((|T|+\delta)(h(|T|+\delta)))$.

In a fashion similar to Lemma 1 , we can see

$$
V|T|(|T|+\delta)^{-1} \rightarrow V\left(1-E_{0}\right)=V \text { (strongly) as } \delta \rightarrow+0 .
$$

Since $V|T|(|T|+\delta)^{-1}$ is contractive, this and (9) imply

$$
T h_{\delta}(|T|) x \rightarrow V(f g)(|T|) x \quad(\delta \rightarrow+0) \text { for } x \in \mathfrak{D}((f g)(|T|)) .
$$

Definition 3. Under the same condition as in Proposition 6, we denote the limit of $T h_{\delta}(|T|)$ by $T h(|T|)$. This is equal to $V(f g)(|T|)$, where $(f g)(t)=t h(t)$.

For non-negative self-adjoint operators $A$ and $B, A \ll B$ means $\mathfrak{D}(B) \subseteq \mathfrak{D}(A)$ and $\|A x\| \leq\|B x\|$ for $x \in \mathfrak{D}(B)$.

If $0 \leq f$ is an operator monotone function defined on $[0, \infty)$, then $f(A) \ll f(B)$ whenever $A \ll B$ (see [3]).

Theorem 7. Let $T$ be a densely defined closed operator, and let $A$ and $B$ be nonnegative self-adjoint operators. Let $f$ and $g$ be arbitrary operator monotone functions defined on $[0, \infty)$, such that $f \geq 0, g \geq 0$. Then $T((f g) / t)(|T|)$ is well defined in the sense of Definition 3. Moreover, if $|T| \ll A,\left|T^{*}\right| \ll B$, then

$$
|(T((f g) / t)(|T|) x, y)| \leq\|f(A) x\|\|g(B) y\|
$$

for every $x \in \mathfrak{D}(A) \cap \mathfrak{D}(f g(|T|))$ and for every $y \in \mathfrak{D}(B)$.

Proof. By Proposition 6 and Definition $3, T((f g) / t)(|T|)$ is well defined and equal to $V(f g)(|T|)$.

The assumption $|T| \ll A,\left|T^{*}\right| \ll B$ implies that $f(|T|) \ll f(A), g\left(\left|T^{*}\right|\right) \ll$ $g(B)$. Suppose

$$
x \in \mathfrak{D}(A) \cap \mathfrak{D}(f g(|T|)) \quad \text { and } \quad y \in \mathfrak{D}(B) .
$$

By Lemma 5, we get

$$
x \in \mathfrak{D}(f(|T|)) \text { and } y \in \mathfrak{D}\left(g\left(\left|T^{*}\right|\right)\right) .
$$

Therefore, $x \in \mathfrak{D}(f(|T|)) \cap \mathfrak{D}(f g(|T|))$ and hence $(f g)(|T|) x=g(|T|) f(|T|) x$. Consequently we get

$$
\begin{aligned}
& |(T h(|T|) x, y)|=|(V(g f)(|T|) x, y)|=|(V g(|T|) f(|T|) x, y)| \\
& =\left|\left(g\left(\left|T^{*}\right|\right) V f(|T|) x, y\right)\right| \leq\|f(|T|) x||\| g\left(\left|T^{*}\right|\right) y\|\leq\| f(A) x\|\| g(B) y \| .
\end{aligned}
$$




\section{REFERENCES}

1. T. Furuta, An extension of the Heinz-Kato theorem, Proc. Amer. Math. Soc. 120 (1994), 785-787. MR 94e:47034

2. E. Heinz, Beiträge zur Strüngstheorie der Spektralzerlegung, Math. Ann. 123 (1951), 415 438. MR 13:471f

3. T. Kato, Notes on some inequalities for linear operators, Math. Ann. 125 (1952), $208-212$. MR 14:766e

4. K. Löwner, Über monotone Matrixfunktionen, Math. Z. 38 (1934), 177 - 216.

5. M. Rosenblum, J. Rovnyak, Hardy classes and operator theory, Oxford University Press, New York, 1985. MR 87e:47001

Department of Mathematics, Fukuoka University of Education, Munakata, Fukuoka, 811-4192, JAPAN

E-mail address: uchiyama@fukuoka-edu.ac.jp 